\title{
INEQUALITIES CONNECTING SOLUTIONS OF CREMONA'S EQUATIONS
}

\author{
G. B. HUFF
}

1. Introduction. Let a complete and regular linear system $\Sigma_{p, d}$ of plane curves of dimension $d$, the genus of the general curve being $p$, be determined by its order $x_{0}$, and its multiplicities $x_{1}, \cdots, x_{\rho}$ at a set of $\rho$ general base points. $x=\left(x_{0} ; x_{1}, \cdots, x_{\rho}\right)$ is called the characteristic of $\Sigma_{p, d}$ and satisfies Cremona's equations:

$$
\begin{array}{r}
x_{0}^{2}-x_{1}^{2}-x_{2}^{2}-\cdots-x_{\rho}^{2} \equiv(x x)=d+p-1, \\
3 x_{0}-x_{1}-x_{2}-\cdots-x_{\rho} \equiv(l x)=d-p+1 .
\end{array}
$$

On the other hand, an integer solution $x$ of (1) may or may not determine a linear system. If an $x$ does determine a $\Sigma_{p, d}$, it is said to be proper. In this definition is included the usual convention that $(0 ;-1,0, \cdots, 0)$ is a proper characteristic of the set of directions at a base point [1].1

If a system $\Sigma_{p, d}$ of characteristic $x$ is subjected to a Cremona transformation $C$ with $F$-points at the base points of $\Sigma, \Sigma \rightarrow \Sigma_{p, d}^{\prime}$ whose characteristic $x^{\prime}$ at the $F$-points of $C^{-1}$ is given by:

$$
L: \begin{array}{r}
x_{0}^{\prime}=(c x) \equiv c_{0} x_{0}-c_{1} x_{1}-c_{2} x_{2}-\cdots-c_{\rho} x_{\rho}, \\
x_{i}^{\prime}=\left(f^{i} x\right) \equiv f_{0}^{i} x_{0}-f_{1}^{i} x_{1}-f_{2}^{i} x_{2}-\cdots-f_{\rho}^{i} x_{\rho}, \\
i=1,2, \cdots, \rho .
\end{array}
$$

Here $c$ is the characteristic of the homaloidal net of $C^{-1}$ and the $f^{i}$ are the characteristics of the $P$-curves of this net. Thus proper characteristics $c$ of $p=0, d=2$ and proper characteristics $f$ of $p=d=0$ play a central role in the theory and will be prominent in this article. The collection of all transformations $L$ for a given $\rho$ forms a group, $G_{\rho}$. $G_{\rho}$ is generated by transformations $L$ for which $c$ is of type (2; $1110 \cdots 0)$, and for any $L \in G_{p}$ the forms $(x x),(l x)$ and $(x y)$ are invariant.

In this paper attention is restricted to characteristics of $x_{0}>0$, and $p \geqq 0$ and $d \geqq 0$. We shall designate this as property $\mathrm{A}$ and obtain inequalities implied by (1) and property $A$. The inequalities are interesting in themselves and lead to a criterion for distinguishing proper characteristics. 1945.

Presented to the Society, November 24, 1945 ; received by the editors November 8 , 1945.

${ }^{1}$ Numbers in brackets refer to the references cited at the end of the paper. 


\section{Inequalities involving the characteristics of homaloidal nets.}

THEOREM 1. If $x$ has property $\mathrm{A}$, then $2 x_{0}-x_{1}-x_{2}-x_{3} \geqq 0$. Moreover, the equals signs hold only for $p=d=0 ; x=(1 ; 110),(1 ; 101)$ or $(1 ; 011)$.

Since $(x x)=d+p-1 \geqq-1$, it may be shown that $x_{0} \geqq x_{i}, i=1, \cdots, \rho$. Indeed, set $x_{i}=x_{0}-a$ in $(x x) \geqq-1$ :

$$
2 a x_{0}-a^{2}-x_{1}^{2}-\cdots-x_{i-1}^{2}-x_{i+1}^{2}-\cdots-x_{\rho}^{2} \geqq-1,
$$

or

$$
a\left(2 x_{0}-a\right) \geqq-1 \text {. }
$$

Since $x_{0}$ is a positive integer, $a$ may not be negative. Thus the integers $a_{1}, a_{2}, a_{3}$ in $x_{1}=x_{0}-a_{1}, x_{2}=x_{0}-a_{2}, x_{3}=x_{0}-a_{3}$ are non-negative. Substituting these in the quadratic relation yields:

$-2 x_{0}^{2}+2 x_{0}\left(a_{1}+a_{2}+a_{3}\right)-a_{1}^{2}-a_{2}^{2}-a_{3}^{2}-x_{4}^{2}-\cdots-x_{\rho}^{2} \geqq-1$.

Now $a_{1}, a_{2}, a_{3}, x_{4}, \cdots, x_{\rho}$ cannot all vanish, for this would imply that $-2 x_{0}^{2} \geqq-1$. Thus:

$$
2 x_{0}\left(a_{1}+a_{2}+a_{3}\right)-2 x_{0}^{2}>-1
$$

or

$$
a_{1}+a_{2}+a_{3}-x_{0}>-1 / 2 x_{0} .
$$

It follows that $a_{1}+a_{2}+a_{3}-x_{0} \geqq 0$ and thus that

$$
2 x_{0}-\left(x_{0}-a_{1}\right)-\left(x_{0}-a_{2}\right)-\left(x_{0}-a_{3}\right) \geqq 0 .
$$

If $x$ is a characteristic with property $\mathrm{A}$ and $2 x_{0}-x_{1}-x_{2}-x_{3}=0$, then the image of $x$ under

$$
\begin{aligned}
x_{0}^{\prime} & =2 x_{0}-x_{1}-x_{2}-x_{3}, \\
\mathrm{~A}_{123}: & x_{i}^{\prime}=x_{i}+\left(x_{0}-x_{1}-x_{2}-x_{3}\right), \quad i=1,2,3, \\
x_{j}^{\prime} & =x_{j}, \quad j=4, \cdots, \rho,
\end{aligned}
$$

has $x_{0}^{\prime}=0$ and satisfies the same Cremona equations. Thus

$$
\begin{aligned}
& -x_{1}^{\prime 2}-x_{2}^{\prime 2}-\cdots-x_{\rho}^{\prime 2}=d+p-1, \\
& -x_{1}^{\prime}-x_{2}^{\prime}-\cdots-x_{\rho}^{\prime}=d-p+1 .
\end{aligned}
$$

This is possible only for $d, p=0,0 ; 1,0$ and 0,1 . A canvass of the cases reveals that $d, p=0,0$ and $x^{\prime}=(0 ;-10 \cdots 0)$ comprise all possibilities. Thus $x=(1 ; 110),(1 ; 101),(1 ; 011)$ are the only values of $x$ for which the equals sign holds. 
Since $(2 ; 1110 \cdots 0)$ is the characteristic of a homaloidal net of conics, the form of the inequality clearly suggests the following generalization:

THEOREM 2. If $x$ has property $\mathrm{A}$ and $c$ is the characteristic of a homaloidal net, then $(c x) \geqq 0$. Moreover, the equals sign holds only for the characteristics of the principal curves of the homaloidal net.

Consider first characteristics $x$ of $p+d>0$. In this case, Theorem 1 asserts that any $x^{\prime}$ obtained from $x$ under $\mathrm{A}_{i j k}$ has $x_{0}^{\prime}>0$. Since $c$ is the characteristic of a homaloidal net, $c$ is the image of $(1 ; 0,0, \cdots, 0)$ under a sequence of transformations of the form $A_{i j k}$. Let $x \rightarrow x^{\prime}$ under the sequence that sends $c \rightarrow c^{\prime}=(1 ; 0, \cdots, 0)$. Since $x_{0}^{\prime}>0$, it follows that $\left(c^{\prime} x^{\prime}\right)>0$. Thus $(c x)>0$, for this bilinear relation is invariant un$\operatorname{der} G_{\rho}$.

If $p=0, d=0$, a modification of the argument is required since in this case $x_{0}^{\prime}$ might vanish under some $\mathrm{A}_{i j k}$. But in this case $x$ is by Theorem 1 a proper characteristic. Thus an improper characteristic $x$ of $p=d=0$ always goes into a characteristic of $x_{0}^{\prime}>0$ under $A_{i j k}$ and the argument above applies. For proper characteristics $x$ of $p=d=0$, it is clear that $(c x) \geqq 0$, else the rational curve would have too many intersections with the homaloidal net. If $(c x)=0, x$ is the characteristic of a rational curve meeting the curves of the net only at the base points, and hence is the characteristic of a principal curve of the net.

\section{Inequalities involving characteristics of rational curves.}

Lemma. If $x$ has property $\mathrm{A}$ and $x^{*}$ denotes the same characteristic with $x_{\rho}$ deleted, then $x^{*}$ has property A.

A simple computation yields for $p^{\prime}, d^{\prime}$ of $x^{*}$ :

$$
d^{\prime}=d+x_{\rho}\left(x_{\rho}+1\right) / 2, \quad p^{\prime}-1=p-1+x_{\rho}\left(x_{\rho}-1\right) / 2 .
$$

Since $x_{\rho}\left(x_{\rho}+1\right) / 2$ and $x_{\rho}\left(x_{\rho}-1\right) / 2$ are non-negative functions of the integer $x_{\rho}$, the conclusion follows.

THEOREM 3. If $x$ has property $\mathrm{A}$ and $p+d>0$, and $f$ is a proper characteristic of $p=d=0$ and $(f x)<0$, then $x_{0}>f_{0}$.

Since $f$ is proper, there is [2] an $L \in G_{\rho}$ such that $\bar{f}=L(f)=(0$; $0, \cdots, 0,-1), \bar{x}=L(x)$ has $\bar{x}_{0}>0$ by Theorem 2 and $(f x)=(\bar{f} \bar{x})<0$. But $(\bar{f} \bar{x})=\bar{x}_{\rho}<0$. Thus $\bar{x}$ may be written in the form

$$
\bar{x}=\bar{x}^{*}+k \bar{f},
$$

where $k$ is a positive integer, and $\bar{x}^{*}$ is $\bar{x}$ with $\bar{x}_{\rho}$ deleted. Now consider the image of $\bar{x}$ under $L^{-1}$. 


$$
L^{-1}(\bar{x})=L^{-1}\left(\bar{x}^{*}+k \bar{f}\right)=L^{-1}\left(\tilde{x}^{*}\right)+k L^{-1}(\bar{f}),
$$

or

$$
x=L^{-1}\left(\bar{x}^{*}\right)+k f .
$$

Now $\bar{x}^{*}$ has $\bar{x}_{0}^{*}>0$, and $p^{\prime}+d^{\prime}>0$ by the lemma. Hence by Theorem 2 its image $\left(\bar{x}^{*}\right)^{\prime}$ has $\left(\bar{x}_{0}^{*}\right)^{\prime}>0$. Since

$$
x_{0}=\left(\bar{x}_{0}^{*}\right)^{\prime}+k f_{0},
$$

it follows that $x_{0}>f_{0}$.

THEOREM 4. If $x$ has property $\mathrm{A}$ and $p+d>0$, and $f$ is a proper charcharacteristic such that $p=d=0$ and $x_{0} \leqq f_{0}$, then $(f x) \geqq 0$.

Theorem 4 follows from Theorem 3 by formal reasoning and offers a generalization of a property of proper characteristics. For if $x$ is a proper characteristic, $(f x) \geqq 0$ follows from the fact that the curves of the system may not have more than $f_{0} x_{0}$ intersections with the irreducible rational curve associated with $f$. The significance of the theorem is that all characteristics $f_{0} \geqq x_{0}>0, p \geqq 0, d \geqq 0, p+d>0$ must enjoy this same property.

There are examples of characteristics with property $\mathrm{A}$ and $p+d>0$ which even have $x_{i}>0, i=1, \cdots, \rho$, for which there is an $f$ of $f_{0}<x_{0}$ such that $(f x)<0$. An early example is $\left(5 ; 3^{2} 1^{6}\right)$ and $\left(1 ; 1^{2} 0^{6}\right)$.

\section{Applications.}

THEOREM 5. Let $x$ be a characteristic of property $\mathrm{A}$ and $p+d>0$, such that $(f x) \geqq 0$ for all proper $f$ of $p=d=0$ and $f_{0}<x_{0}$; then $x_{i} \geqq 0$ and, moreover, if $x^{\prime}$ is the image of $x$ under any $L \in G_{\rho}$, then $x_{0}^{\prime}>0$ and $x_{i}^{\prime} \geqq 0$ for $i=1, \cdots, \rho$.

Since $\bar{f}=\left(0 ; 0^{p-1}-1\right)$ is a proper $f$ of $f_{0}=0<x_{0}$ and $(\bar{f} x) \geqq 0$, it follows that $x_{i} \geqq 0$. By Theorem $4,(f x) \geqq 0$ for all proper $f, p=d=0$ of $f_{0} \geqq x_{0}$. Then $(f x) \geqq 0$ for all proper $f$. These characteristics $f$ are simply permuted by any $L \in G_{\rho}$. Thus if $x^{\prime}=L(x)$, it follows that $\left(f x^{\prime}\right) \geqq 0$ for all proper $f$. Since these include $\left(0 ; 0^{\rho-1}-1\right)$, it follows as before that $x_{i}^{\prime} \geqq 0$. Theorem 2 asserts that $x_{0}^{\prime}>0$.

The following important result is now easily established:

THEOREM 6. Let $c$ be a solution of (1) for $p=0, d=2, c_{0}>0$ such that $(f c) \geqq 0$ for all proper $f$ of $p=d=0$ and $f_{0}<c_{0}$, then $c$ is the characteristic of a homaloidal net.

As before, $c_{i} \geqq 0$ and it is known [3] that in such a case $c_{0}-c_{1}-c_{2}$ $-c_{3}<0$ if $c_{1}, c_{2}, c_{3}$ are the greatest of the numbers $c_{i}$ and $c_{0}>1$. Thus 
under $\mathrm{A}_{123}, c \rightarrow c^{\prime}$ of $c_{0}^{\prime}<c_{0}$ and by Theorem $5, c_{i}^{\prime} \geqq 0, i=1,2, \cdots, \rho$. Thus this reduction may be continued until $c_{0}^{\prime \prime}=1$, in which case $c^{\prime \prime}=(1 ; 0, \cdots, 0)$. Under the given hypotheses, $c$ is then the image of $(1 ; 0, \cdots, 0)$ under some $L \in G_{\rho}$ and must be proper.

This result has been conjectured much earlier and indeed was proved [4] by the writer, but the proof given on that occasion was quite elusive and unsatisfactory. Fragmentary results indicate that Theorem 5 has other important applications to cases where a generalization of Noether's inequality is possible. It would be desirable to avoid the restriction $p+d>0$. It is possible that Theorem $5 \mathrm{might}$ still be true if one removed this restriction and added at the end of the theorem "or else $x^{\prime}$ is of the type $(0 ; 0, \cdots, 0,-1)$."

\section{REFERENCES}

1. A. B. Coble, Cremona's diophantine equations, Amer. J. Math. vol. 56 (1934) pp. 459-461. See especially p. 461.

2. J. L. Coolidge, Algebraic plane curves, 1931, p. 399.

3. Max Noether, Ueber Flachen welche Schaaren rationaler Curven besitzen, Math. Ann. vol. 3 (1871) p. 167.

4. G. B. Huff, A sufficient condition that a $C$-characteristic be geometric, Proc. Nat. Acad. Sci. U.S.A. vol. 29 (1943) p. 198.

UNIVERSITY OF TEXAS 